\title{
UNA NOTA SOBRE LA PRODUCCION AGRARIA DE ANDALUCIA ORIENTAL EN 1874-1914 *
}

JOSE MORILLA CRITZ Universidad de Alcalá de Henares

No creo exagerado decir que esta obra de Jiménez Blanco constituye, hoy por hoy, el trabajo más importante sobre la agricultura de Andalucía Oriental en el siglo XIX. Por otra parte, son dignas de destacar sus exhaustivas exposiciones del estado de la cuestión en cada subsector y las pormenorizadas explicaciones sobre la situación y utilización de las fuentes. Esto es particularmente útil en una parcela de la historia de Andalucía, caracterizada por la multiplicación de trabajos de ámbito reducido. Todo ello creo que justifica una nota crítica de los dos volúmenes en que se divide el trabajo, más amplia y detallada que lo que permite una simple recensión.

El hilo conductor principal de la investigación de Jiménez Blanco en este caso es el análisis de la respuesta que dieron agricultores y empresarios de la industria agroalimentaria de Andalucía Oriental al cambio de las circunstancias de los mercados de sus productos. Una fuente de incitaciones a los cambios de los sistemas productivos venía de los mercados exteriores y se puede sintetizar en los componentes de la «gran depresión» del último cuarto del siglo xix. Otra incitación provenía de los cambios en la demanda interior de productos alimenticios.

Por supuesto que, actuando simultáneamente con las circunstancias citadas, estuvo la acción del Estado, que tampoco olvida Jiménez Blanco. La política arancelaria modificaba las condiciones de competencia exterior. Otras medidas de política económica, como las orientadas a redefinir los principios de la propiedad y el uso de los montes, condicionaron, sobre todo, las respuestas del sector ganadero.

Los cereales y leguminosas, la ganadería y los montes públicos

Los cereales y leguminosas, los usos de los montes públicos y la ganadería tuvieron en Andalucía Oriental un comportamiento muy parecido al de esos

* J. I. Jiménez Blanco, La producción agraria de Andalucia Oriental, 1874-1914, Ed. Universidad Complutense de Madrid, Servicio de Reprografia, Madrid, 1986. 
mismos sectores de la agricultura nacional, salvando algunos desfases temporales y variedades provinciales:

- La producción de cereales y leguminosas se incrementó notablemente, entre 1886 y 1930-1935, mediante un uso más intensivo que hasta entonces de la superficie cultivada y, también, mediante la elevación de los rendimientos en la superficie sembrada. Esto fue posible gracias al empleo creciente de abonos químicos y mediante la sustitución de los aperos de labranza tradicionales (sobre todo arados romanos) por otros más avanzados (arados de reja y vertedera).

- La ganadería de AOR, con leves diferencias con respecto al resto de España, comenzó una recuperación, tras la profunda crisis de la segunda mitad del siglo XIx, hacia 1895. En 1925, AOR había acortado distancias, en cuanto a la producción pecuaria, con el resto de España. El crecimiento ganadero vino impulsado por el de la demanda de proteínas ligado al aumento del nivel de vida, a la urbanización de la población y a la ventaja comparativa de orientar la producción interior de subsistencias a los productos animales, dada la baratura de los cereales en el mercado internacional. No obstante, y a pesar de una orientación de la ganadería de AOR hacia el ganado-renta (como en toda España), las condiciones naturales de la subregión determinaron una menor importancia del vacuno y una mayor importancia relativa del cabrío, de porcino y de las aves.

- El sistema cereal respondió al incremento ganadero, orientándose, al igual que en el resto de España, aunque con alguna lentitud, a la producción de piensos en detrimento de alimento humano directo.

- El incremento de la producción de cereales-pienso, el aprovechamiento de algunos esquilmos como forraje y el empleo de desperdicios de la alimentación humana no fueron, a pesar de todo, suficientes para sostener el crecimiento de la cabaña ganadera y, dada la reducción de los barbechos y eriales, una buena parte de la alimentación de esa ganadería en crecimiento hubo de resolverse con la intensificación de los aprovechamientos de pastos espontáneos y montes ante las dificultades de la región para el desarrollo de praderas artificiales, salvo en zonas de vega muy localizadas. Las crisis de algunos cultivos tradicionales en algunas provincias (como la viticultura de los montes periféricos) y, sobre todo, un mejor y más ordenado aprovechamiento de los montes públicos permitieron disponer a la ganadería del complemento alimentario no cubierto por la producción cerealera. Con todo ello, AOR consiguió un crecimiento de la producción pecuaria superior al de la media nacional. 


\section{El olivar}

La evolución de este sector estuvo condicionada, sobre todo, por la situación de los mercados exteriores de aceites. Según Jiménez Blanco, que en esto refleja lo ya estudiado por otros investigadores como Zambrana, la depresión que se manifestó en el olivar de AOR entre 1890 y 1911 estuvo motivada sobre todo por la paulatina reducción de la demanda de aceite de oliva para usos industriales a medida que se iba consolidando la de aceites minerales y de semillas, más aptos y baratos para dichos usos.

La recuperación del sector se empezó a manifestar en las cifras de producción de 1912, pero, teniendo en cuenta el tiempo que ha de transcurrir entre la inversión en plantaciones y su expresión en la producción en este tipo de cultivo, los cambios que la hicieron posible se pusieron en marcha entre 1897 y 1906, e incluso antes. Esos cambios fueron muy importantes, porque el sector olivarero de AOR que se desarrolló desde entonces poco tenía que ver con el que estaba en crisis. Dos circunstancias englobaron los cambios referidos:

a) La especialización territorial: La provincia de Jaén se convirtió desde principios del siglo $\mathrm{xx}$ en el principal núcleo olivarero de la subregión y de España ( 80 por 100 de la producción de AOR, 44,3 por 100 de Andalucía y 24,5 por 100 de España). humano.

b) La especialización en la producción de aceite de oliva para consumo

Las razones de la primera de estas especializaciones, levemente tratadas por Jiménez Blanco, han de encontrarse en las ventajas comparativas de este cultivo en Jaén en relación a las otras provincias. Estas ventajas eran absolutas con respecto a Almería, dadas las características climatológicas de la misma, y relativas respecto a Granada y Málaga, dado, seguramente, el menor coste de oportunidad del olivar en Jaén frente a las posibilidades de otros cultivos, también en expansión en las otras dos provincias.

Las mejoras en los sistemas de cultivo del olivar se extendieron por Andalucía Oriental claramente desde 1900 , especialmente en las plantaciones de mayores dimensiones. Consistieron en la selección de las variedades de árboles más aptas para cada terreno y de mayor capacidad productiva, utilización de abonos y plaguicidas, eliminación del cultivo asociado, aplicación de sistemas adecuados de poda, mayor cuidado y pulcritud en las labores de *vareo* y recogida del fruto del suelo y limpieza del fruto antes de su entrada en la almazara.

En el proceso industrial de obtención del aceite hubo una auténtica re- 
volución en Jaén, provincia en la que, a partir de 1906-1910, el rendimiento por QM. de aceituna superó a la media nacional y llegó, hacia 1930, a rendimientos similares a los de hoy día. Pero a ello acompañó también una progresiva mejora en la calidad del aceite que, gracias a ella, pudo ir introduciéndose en el mercado de grasas para consumo humano. Los términos técnicos de estas mejoras se encontraron, por un lado, en el incremento de la potencia y rapidez de la molinería y prensado y, por otro, en la transformación radical de los sistemas de decantación, filtrado, transporte, etc., con lo que se eliminaron todos los elementos tóxicos y de mal olor y sabor, que impedían anteriormente la utilización del aceite andaluz para la alimentación.

\section{El viñedo}

El estudio del sector vitícola en Andalucía Oriental en los tres últimos decenios del siglo $\mathrm{xIX}$ y en los dos primeros del $\mathrm{xx}$ es muy importante, porque su evolución en tales años y su relación con la «decadencia» de los inicios industriales de la subregión ha levantado, desde hace trece años, una cierta polémica entre los historiadores económicos.

Las conclusiones de Jiménez Blanco sobre cada uno de los subsectores de la viticultura de Andalucía Oriental en el período estudiado se pueden resumir de la siguiente forma:

Vino (por lo que hace a Málaga, principalmente): Era un subsector de la viticultura de AOR en decadencia desde comienzos del siglo xIx, pero que asistió a una expansión, al igual que en toda España, entre 1873 y 1886, por "distintos motivos" que en el resto de la nación, aunque no se considera en condiciones de señalar cuáles pudieron ser. Entre 1886 y 1904 decayó, no obstante, la producción del principal vino exportable (el de Málaga), como consecuencia de la filoxera, y se recuperó desde el último año hasta la Primera Guerra Mundial.

Pasas (también en Málaga): La documentación y los razonamientos de Jiménez Blanco aportan nuevas evidencias a la tesis, mantenida por una parte de los que hasta hoy hemos participado en la polémica antes mencionada, de que el subsector pasero de AOR comenzó una fuerte crisis antes de la aparición de la filoxera en la zona, como consecuencia de la pérdida paulatina de sus mercados exteriores y de que esta pérdida fue una consecuencia del fenómeno general de los cambios impulsados por la irrupción de la agricultura ultramarina en el mercado mundial.

Uva de embarque (de Almería, fundamentalmente): La evolución de esta parcela de la viticultura fue el contrapunto a la crisis del subsector pasero malagueño: la demanda exterior desde mediados del siglo $\mathrm{xIx}$, en unión de 
las ventajas de tipo natural de la provincia más oriental, hicieron que los parrales se extendieran extraordinariamente por todas las zonas aptas a los mismos, a pesar de la invasión filoxérica. Después, espoleada la producción por la creciente demanda, el subsector asistió a una mejora en los sistemas de cultivo, tratamiento del fruto, envasado y comercialización.

El problema de la filoxera: De acuerdo a lo analizado para cada subsector e incorporando lo aportado por las más recientes investigaciones, Jiménez Blanco relativiza, como otros autores, el papel que de forma directa desempeñó la filoxera en la crisis y transformación de la viticultura de AOR.

En los esquilmos para los que las indicaciones de los mercados exteriores eran favorables, la lucha contra la plaga fue escasa; lo contrario ocurrió con los esquilmos para los que había favorables expectativas.

La evolución de los rendimientos: Jiménez Blanco intenta penetrar en el conocimiento de esta importante variable mediante los datos procedentes de los Informes de los ingenieros agrónomos provinciales, que, por deficiencias de las series, utiliza para analizar solamente lo ocurrido en el tramo 19081922.

Llega a la conclusión de que los rendimientos medios de la viticultura de Andalucía Oriental tras la crisis filoxérica y de los mercados (1874-1908), a pesar de haberse incrementado, eran inferiores a los de la media nacional y bastante más bajos que los de otra zona mediterránea como Cataluña, registrando, además, un deterioro a lo largo del segundo decenio del siglo $\mathbf{x x}$, sobre todo en lo que hacía a las cepas para vinificación. El incremento leve de los rendimientos en las cepas para pasa en Málaga se debió a una reducción de la superficie dedicada a este cultivo. En la uva de Almería hubo fuertes crecimientos de los rendimientos en los primeros años, que fueron luego descendiendo. En conjunto, todo indicaría, según J. Blanco, unos sistemas de cultivo y técnicas de elaboración que dejaban bastante que desear para las posibilidades de la época.

Creo, no obstante, que el estudio de los rendimientos vitícolas de Jiménez Blanco tienen algunas deficiencias debidas, principalmente, a dos cuestiones:

a) En una viticultura con una transformación tan intensa como la de Andalucía Oriental, en la que predominó sobre todo el cambio de estructura de la producción final, el término significativo de comparación ha de ser, en todo caso, la producción total de uva en relación a la superficie de cepas.

b) Hay datos alternativos fiables que indican que las cifras de producción de uva de los ingenieros agrónomos provinciales, al menos para AOR, están extraordinariamente subestimadas. 
En consecuencia, parece ser que la productividad debía de ser considerablemente más alta, y que ello era la consecuencia de la «revolución» habida, sobre todo, en el subsector de la uva para embarque.

\section{Las plantas industriales}

Los comienzos del cultivo de la remolacha y de la industria azucareraremolachera en Granada han sido no hace mucho estudiados magníficamente por Martín Rodríguez, y por ello el trabajo de Jiménez Blanco es, en gran medida, y así se reconoce por el autor, deudor, en cuanto a información y conclusiones, del libro de aquél. No obstante, Jiménez Blanco ha incorporado nueva información, procedente en su mayor parte de fuentes fiscales, y ha tratado el subsector cañero con algo más de detenimiento y relación con el remolachero.

La producción azucarera-remolachera europea y la discriminación en el mercado español a favor de los azúcares de las colonias, que mejoró entre 1884 y 1898, establecieron desde el primer año condiciones de competencia muy duras para la caña de la zona subtropical española, que detuvo la tendencia creciente que manifestaba su producción desde mediados de siglo, eli. minándose los cosecheros marginales. La pérdida de las últimas colonias y el proteccionismo generalizado desde entonces para el sector azucarero permitieron un último esplendor del azúcar de caña granadino entre aquella fecha y 1905. No obstante, en los años en que la caña había llegado al máximo de superficie cultivada y estaba sufriendo, a la vez, la competencia del azúcar colonial (1880-1898), surgió y comenzó a desarrollarse en la vega granadina el cultivo y la industria de la remolacha azucarera. En las circunstancias expuestas, el surgimiento de esta nueva actividad en Granada fue la consecuencia de dos tipos de causas:

a) La combinación de las políticas arancelarias y fiscales, que fueron siempre, de derecho o de hecho, instrumentos de protección muy adecuados para la producción remolachera nacional frente a la extranjera, que se unían a las ventajas que, en cualquier caso, tenía la remolacha sobre la caña. Una vez perdidas las colonias, la reforma fiscal de Villaverde, en 1899, permitió una protección manifiesta de derecho para la industria remolachera naciente.

b) La necesidad de los cultivadores granadinos de encontrar un cultivo que sustituyera, en la rotación tradicional de la vega, a las producciones de cáñamo y lino, en crisis desde comienzos del siglo XIX.

El cultivo de la remolacha y la industria azucarera se extendieron rápidamente por la vega de Granada hasta 1891, y desde entonces se incorporaron 
a esas actividades otras provincias, principalmente Málaga y Zaragoza. A partir de ese momento, dos circunstancias, actuando conjuntamente, perjudicaron a la provincia granadina en la explotación azucarera-remolachera y fueron provocando una reducción del cultivo:

1. Un creciente exceso de producción nacional, dado el nivel de consumo azucarero existente.

2. Las desventajas técnicas y organizativas de haber sido la vega granadina un first comers en este tipo de industria.

Por otra parte, Andalucía Oriental acabó recibiendo también parte del impacto negativo del desarrollo de la industria azucarera-remolachera, al abarcar en su seno todo el cultivo y transformación nacional de la caña de azúcar. La competencia remolachera obligó a una reducción de la producción de aquel subsector entre 1905 y 1920, año este último en el que, limitado el cultivo a las tierras más adecuadas (vega de Motril), concentrada la industria y establecidas condiciones más desfavorables para los agricultores, restableció parcialmente una situación competitiva.

En cualquier caso, la irrupción de la remolacha y la industria derivada en la vega de Granada fue de gran trascendencia para esta provincia, puesto que en tal comarca se produjeron los siguientes efectos:

- Se modificó el ciclo tradicional de las cosechas, reduciéndose notablemente la superficie total de barbecho.

- Obligó a importantes transformaciones técnicas, desde el abonado a la maquinaria, de las que se beneficiaron todos los cultivos.

- Con todo ello, se incrementaron, en su conjunto, los rendimientos y las rentas de los agricultores.

Finalmente, Jiménez Blanco trae a colación, como efecto indirecto de la evolución del cultivo remolachero de la vega granadina, el desarrollo, desde 1923, del cultivo del tabaco, puesto que su aparición en Granada fue la consecuencia de una nueva necesidad de la rotación de cultivos de la vega tras la crisis de la industria azucarera-remolachera de la provincia.

\section{Otros cultivos}

El estudio pormenorizado de la agricultura de Andalucía Oriental de Jiménez Blanco termina con la consideración de tres cultivos que desempeñaron importantes papeles en la transformación del agro de la subregión en los primeros decenios del siglo xx: la patata, el almendro y los cítricos. De todos 
ellos, así como de la horticultura en general (que trata levemente), las fuentes cuantitativas directas son escasas, si exceptuamos los datos de comercio exterior.

La patata colaboró, en la vega de Granada principalmente, a solventar parte del problema de la alimentación de las clases populares y, también, a completar la nueva rotación de cultivos impuesta por la introducción de cultivos como la remolacha y el tabaco. Impulsó también el perfeccionamiento técnico de la agricultura en todas las comarcas por las que se extendió, al ser una planta que obligaba al empleo de abonos inorgánicos. En el litoral, por otra parte, su establecimiento apoyó la recuperación de la economía exportadora, que había sufrido el trauma de la crisis vitícola, pues se inició desde entonces, sobre todo en Almeria, la producción de patata temprana para mercados exteriores.

Las plantaciones de almendros y naranjos estuvieron en expansión desde el comienzo de la crisis vitícola hasta los años treinta del siglo $\mathrm{xx}$, ambos en la provincia de Málaga y los segundos también en la de Almería. El impulso para esta expansión vino dado por la demanda exterior y, en conjunto, constituyeron también una alternativa a las crisis de la viticultura tradicional.

Con respecto a las plantas hortícolas, el estudio impresionista que Jiménez Blanco hace para 1914 le permite afirmar que en el interior de la subregión aún no se había salido en estos cultivos de unas prácticas tradicionales, destinándose la producción al consumo en mercados locales y comarçales, mientras que en el litoral comenzó lentamente el desarrollo de cultivos tempranos cuyos frutos se destinaban, sobre todo en Almería, a mercados extranjeros.

\section{Visión global de la agricultura de $A O R$ a comienzos del siglo $X X$}

Jiménez Blanco termina su obra con un estudio sintético de la producción y productividad agrarias de AOR entre 1900 y 1931, que, en parte, ya conocíamos gracias a un opúsculo suyo de 1985. De sus datos deduce, como nota más significativa, que la agricultura de la subregión asistió en los tres primeros decenios del siglo $\mathrm{xx}$ a una expansión $\mathrm{y}$, al mismo tiempo, a una transformación productiva, que tuvo como principales características las siguientes:

1. Creció la superficie cultivada en una proporción superior a la media nacional, y ese crecimiento se hizo a costa de barbechos, montes y eriales. Proporcionalmente, los cultivos que se beneficiaron de ese crecimiento de la superficie en mayor medida fueron los arbóreos (almendros, olivares, cítricos), seguidos de las plantas de regadío (remolacha y patatas), y participaron en menor medida el viñedo $y$, sobre todo, cereales y leguminosas. 
2. El producto agrario durante los tres decenios, a precios constantes de 1910, registró un crecimiento en el que los puntos más significativos fueron:

a) Un mayor incremento relativo del producto pecuario (superior a la media nacional) que el del agrícola y una fuerte reducción (más intensa que la media nacional) del producto de montes, dehesas y pastos.

b) Dentro del producto agrícola, el alza, en consonancia con la extensión respectiva de los cultivos, se manifestó principalmente en las plantas arbustivas, seguidas de las intensivas de regadio y el viñedo, siendo casi imperceptible el crecimiento del producto correspondiente a cereales y leguminosas.

3. La productividad de la tierra, en términos monetarios constantes, no cejó de progresar en esos años en los cultivos arbustivos, en los de regadío y en el viñedo, y se estancó en cereales y leguminosas.

Todo lo anterior indica que Andalucía Oriental se fue especializando en esos tres decenios, cada vez más, en la ganadería y en los cultivos arbóreos y de regadío, que esa especialización estuvo en consonancia con un aprovechamiento más adecuado que en épocas anteriores de las características naturales de la zona y que las innovaciones tecnológicas se concentraron en los sectores más adaptados a aquellas condiciones naturales.

El resultado global de esa transformación, en comparación al resto de España, fue que el crecimiento del producto agrario por persona de la subregión fue superior a la media nacional y el de la productividad por persona activa masculina similar a aquélla, pero, no obstante, en 1931, AOR todavía tenía niveles de tales magnitudes inferiores a la media nacional, porque había partido, en 1900, de niveles inferiores.

En conjunto, el anterior análisis permite afirmar que el sector primario de Andalucía Oriental progresó notablemente en los tres primeros decenios del siglo $\mathrm{xx}$, aprovechando las ventajas comparativas de la subregión de forma más eficiente que en épocas anteriores y siguiendo las indicaciones del mercado. En consecuencia, concluye Jiménez Blanco, la agricultura en Andalucía Oriental no sólo no estuvo estancada, como a veces se ha manifestado, sino que mostró entonces un notable dinamismo para adaptarse, casi siempre con éxito, a las condiciones cambiantes de los mercados.

\section{Consideraciones finales $y$ algunas criticas}

La obra de Jiménez Blanco, la más extensa y sólida hasta ahora sobre el conjunto de la viticultura de Andalucía Oriental a finales del xIx y principios 
del xx es, aparte de todo lo expuesto en las líneas que anteceden, el más detallado y documentado alegato a favor de la línea argumental que Bernal ha calificado de «ausencia de inmovilismo y atraso» y de "afirmación de la modernización y cambio», con la que se puede estar enteramente de acuerdo o no, pero que, como el propio trabajo de Jiménez Blanco demuestra, tiene bases en las que sustentarse. Ello al margen de que, posiblemente, empeorara, en términos sociales, la distribución de la renta del sector y el proceso de ajuste "productivista» a la crisis finisecular produjera efectos dramáticos para los agricultores tradicionales.

Aparte de algunas críticas que hemos hecho al trabajo líneas arriba, hay, según mi opinión, algunas (pocas) deficiencias que llevan a Jiménez Blanco a daducir en algún caso conclusiones erróneas. Por ejemplo, al tratar de la evolución tecnológica del sistema cereal, considera como signo de atraso en la provincia de Granada, por contraste a las otras de la región, la elevada proporción de arados romanos, sin caer en la cuenta de que el desarrollo de cultivos «modernos», como la remolacha y la patata (que precisamente se implantan extensamente en Granada), iba aparejada hasta la era de los tractores, y sobre todo de la perfección de sus accesorios, a la necesidad de volver a emplear el arado romano, pues era el único con el que se podian arrancar los tubérculos sin dañarlos.

En la investigación del rendimiento de carne por animal considero que no hay que ser tan pesimista como Jiménez Blanco sobre la inexistencia de fuentes, porque los estadillos de mataderos locales son bastante abundantes, publicados incluso a veces en los Boletines Provinciales.

Finalmente, creo que no es suficiente utilizar como muestra de la evolución de los cítricos (alternativa a la viticultura) la suerte del naranjo exclusivamente. La tendencia depresiva de la producción y negocio de la naranja en Málaga, desde 1880 , se ha de compensar con el crecimiento, por el contrario, de otros círricos como el limonero y variedades «amargas» de naranja.

En cualquier caso, estas y otras deficiencias no son de gran importancia en una obra que marca un hito en la historia agraria de Andalucía Oriental. 